\title{
Mass of Spiral Galaxies by Means of a Maximum Disc Model
}

\author{
Jerson I. Reina ${ }^{1,2, *}$, Framsol López-Suspes ${ }^{1,2, * *}$, Guillermo A. González ${ }^{2, * * *}$
}

\section{Edited by}

Juan Carlos Salcedo-Reyes

(salcedo.juan@javeriana.edu.co)

1. Departamento de Ciencias Básicas, Universidad Santo Tomás,

Bucaramanga, Colombia.

2. Escuela de Física, Universidad Industrial de Santander, Bucaramanga, Colombia.

*jerson.reina@ustabuca.edu.co

** framsol.lopez@ustabuca.edu.co

*** guillermo.gonzalez@saber.uis.edu.co

Received: 22-09-2017

Accepted: 31-05-2018

Published on line: 30-07-2018

Citation: Reina JI, López-Suspes F, González GA. Mass of Spiral Galaxies by Means of a Maximum Disc Model, Universitas Scientiarum, 23 (2): 191-218, 2018. doi: 10.11144/Javeriana.SC23-2.mosg

Funding:

N.A.

Electronic supplementary material: N.A.

OPEN ACCESS

\begin{abstract}
Maximum disc mass models for a set of spiral galaxies from the Ursa Major Cluster are presented. We construct the models using the Hunther method and the particular solutions are chosen in such a way that the circular velocities are adjusted very accurately to the observed rotation curves of some specific spiral galaxies. Under the maximum disc hypothesis, we consider that the rotation curves of the analyzed galaxies can be modeled with only the contribution of the disc. This implies that it is not necessary to consider the contribution ot the dark matter halo in the inner part of the spiral. In this way, the models reproduce the global behavior of the rotation curves in the great majority of galaxies. Producing good adjustments to calculate the total mass of these galaxies, and yielding values of the order of $10^{10} \mathscr{M}_{\odot}$. Based on the vertical stability criterion presented by Viera \& Ramos-caro (2016), we find that all the galaxies analyzed present a vertically stable behavior. On the other hand, from the analysis of the epicyclic frequency we find that all the models exhibit mainly a radial stable behaviour except at the edge of the disc.
\end{abstract}

Keywords: Potential Theory; Stellar Dynamics; Galactic Mass.

\section{Introduction}

One of the greatest challenges of modern astronomy is the determination of the origin, nature and distribution of matter in its visible and dark forms. Regardless of whether the content of matter in a spiral galaxy is luminous or not, the gravity is the only force that affects all forms of matter, whatever its properties and conditions. So that by probing the effects of gravity through its dynamic influence, we can reveal the whole material content of a system. Currently one of the best tools available to analyze the material content of a spiral galaxy is the rotation curve (Binney \& Tremaine, 2008; Leblanc, 2010; Rubin et al. 1985), which shows how the stars in a galaxy rotate around the center in approximately circular orbits (Oort, 1927), with a velocity that varies only under the effects of the galaxy's own gravity. 
In order to determine the mass distribution of a particular spiral galaxy, it is necessary to propose a model in such a way that it adjusts with the data of the rotation curve of the galaxy analyzed. Taking into account that each of the components of a spiral galaxy contributes to the rotation curve, there are in the literature several mass models that include one to several components depending on the particular situation to be modeled (Sofue et al. 2009). For example, Bajkova \& Bobylev (2016) fitted a three component (bulge, disc, halo) model to the rotation velocities of Galactic objects known from observations in the range of Galactocentric distances $R$ from 0 to $200 \mathrm{kpc}$. Recently, Gonzáles \& Reina (2016) presented a family of analytical potentials for flat galaxies with spheroidal halos characterized by superposition of two independent components: a potential associated with the thin disc and a potential associated with spheroidal halo. Kalnajs (1983) and Van Albada et al. (1985), among other authors, showed that the combination of a central bulb surrounded by an exponential disk produces a flat velocity curve with a reasonable stellar mass-to-light ratio.

From the maximum disc hypothesis (Palunas \& Williams, 2000), the mass of the galactic disc in a spiral galaxy is accepted to be large enough, so that it is consistent with the rotation curve of the galaxy. In this way, it is not necessary to consider the contribution of the dark matter halo in the inner part to the spiral. Thus, some authors have shown that it is possible to obtain galactic models taking into account only the disc component, without having to include a halo of matter, modeling with high precision rotation curves in the optical region and according to physically reasonable mass density profiles (Kalnajs, 1983; Kent, 1986; Gonzáles et al. 2010).

In particular, Gonzáles et al. (2010) obtained finite thin disc models for four galaxies in the Ursa Major cluster: NGC3877, NGC3917, NGC3949 and NGC4010. The models were obtained through the Hunter method (Hunter, 1963) and the particular solutions were chosen in such a way that the circular velocities were adjusted very precisely with the data of some rotation curves taken from Verheijen \& Sancisi (2001). The obtained models are vertically unstable under the criterion of vertical frequency stability (Binney \& Tremaine, 2008). However, recently Viera \& Ramos-caro (2016) found that in each thin disc galactic model, all circular equatorial orbits within the matter distribution are vertically stable, which reverses the instability argument of the models of Gonzáles et al. (2010).

In order to illustrate the applicability of the procedure proposed by Viera \& Ramos-caro (2016), we are going to apply the methodology proposed in Gonzáles et al. (2010), this time analyzing the extension of the model to all galaxies in the sample. Verheijen \& Sancisi,(2001). In this way we pretend to 
remark that the formalism proposed by Gonzáles et al. (2010) is a powerful method for obtaining stable maximum discs that describe the optical region of many galaxies.

For this purpose, we initially summarize the most remarkable aspects of the construction of the maximum disc models proposed by Gonzáles et al. (2010). We then analyze the formalism developed by Viera \& Ramos-caro (2016) and the way in which the analysis of the stability of the equatorial orbits must take into account the discontinuity in the partial derivative of the potential with respect to $z$. Later, we adjust the model to the data of the observed rotation curves of all the galaxies reported in Verheijen \& Sancisi (2001) and, finally, we present a discussion of the obtained results.

\section{Thin Disc Mass Models}

As it was developed by Gonzáles \& Reina (2006), the starting point to construct finite, axially symmetric disc models is to solve the Laplace equation, which in cylindrical coordinates is written as

$$
\frac{\partial^{2} \Phi}{\partial R^{2}}+\frac{1}{R} \frac{\partial \Phi}{\partial R}+\frac{\partial^{2} \Phi}{\partial z^{2}}=0
$$

As we will present in the next section, in order to apply the vertical stability criterion for circular orbits, recently developed by Viera \& Ramos-caro (2016), we must consider that the reflection symmetry

$$
\Phi(R, z)=\Phi(R,-z),
$$

implies that the gravitational potential depends only on the absolute value $|z|$ of $z$. so we can rewrite the normal derivative as

$$
\frac{\partial \Phi}{\partial z}=\frac{\partial \Phi}{\partial|z|} \frac{\partial|z|}{\partial z}=[2 \Theta(z)-1] \frac{\partial \Phi}{\partial|z|},
$$

where $\Theta$ is the Heaviside step-function. Thus, the potential $\Phi$ in these models is a smooth function of $|z|$ with a discontinuity in the $z$ derivative at the plane $z=0$.

Given a gravitational potential $\Phi(R, z)$ that satisfies the above considerations, the circular velocity $v_{c}(R)$ can be obtained from the gravitational potential through the relation

$$
v_{c}^{2}(R)=\left.R \frac{\partial \Phi}{\partial R}\right|_{z=0}
$$

Besides, by means of the Gauss Theorem and the equation (3), we can express the surface distribution of matter as

$$
\Sigma(R)=\left.\frac{1}{2 \pi G} \frac{\partial \Phi}{\partial|z|}\right|_{z=0},
$$


which must be adjusted to the boundary conditions

$$
\begin{aligned}
& \left.\frac{\partial \Phi}{\partial|z|}\right|_{z=0} \neq 0 ; \quad R \leq a, \\
& \left.\frac{\partial \Phi}{\partial|z|}\right|_{z=0}=0 ; R>a,
\end{aligned}
$$

in order to obtain a discoidal distribution of matter restricted to the plane $z=0$ and with a radius $R$ in the interval $0<R<a$.

As it was developed by Gonzáles \& Reina (2006) and Gonzáles et al. (2010), a gravitational potential satisfying the conditions mentioned above and describing a discoidal distribution of axially symmetric matter has the form (Bateman, 1944)

$$
\Phi(\xi, \eta)=-\sum_{n=0}^{\infty} C_{2 n} q_{2 n}(\xi) P_{2 n}(\eta) .
$$

Here $P_{n}(\eta)$ are the usual Legendre polynomials and $q_{n}(\xi)=i^{n+1} Q_{n}(i \xi)$, being $Q_{n}(z)$ the Legendre functions of the second kind. The spheroidal oblate coordinates $(\xi, \eta)$, with $0 \leq \xi<\infty$ and $-1 \leq \eta<1$, are related to the usual cylindrical coordinates $(R, z)$ through the relations

$$
\begin{aligned}
R & =a \sqrt{\left(1+\xi^{2}\right)\left(1-\eta^{2}\right)} \\
z & =a \xi \eta
\end{aligned}
$$

in such a way that the disc are located at $\xi=0, \eta=\sqrt{1-R^{2} / a^{2}}$. Finally the $C_{2 n}$ are arbitrary constants, which are chosen properly in order to adjust the circular velocity of the model and the rotation curve of some particular galaxy.

With this gravitational potential, and using the equations (4) and (5) we have that the circular velocity and the surface mass density can be written respectively as

$$
v_{c}^{2}(\widetilde{R})=\frac{\widetilde{R}^{2}}{\eta} \sum_{n=1}^{\infty} C_{2 n} q_{2 n}(0) P_{2 n}^{\prime}(\eta)
$$

and

$$
\Sigma(\widetilde{R})=\frac{1}{2 \pi a G \eta} \sum_{n=0}^{\infty} C_{2 n}(2 n+1) q_{2 n+1}(0) P_{2 n}(\eta),
$$

where $\widetilde{R}=R / a$. By integrating over the total area of the disc, we find the expression

$$
\frac{\mathscr{M} G}{a}=C_{0}
$$


which we will use to determine the total mass of each galaxy $\mathscr{M}$. In this way, we can see from (7), (9) and (10) that the whole model is completely defined in terms of constants $C_{2 n}$, which will be determined by fitting (9) with the observational data associated with the rotation curve of a particular galaxy.

\section{A Family of Particular Models}

In this section we show how from (9) we can adjust the circular velocity of the model with the observed data for a given galaxy in order to construct particular models of thin disks. To this end, we must take into account that the sum must be limited to a finite number of terms. This implies that $C_{2 n}=0$ for $n>m$, with $m$ a positive integer, which represents the number of parameters necessary to obtain the best fit. Replacing the derivatives of the Legendre polynomials in (9) we can rewrite the circular velocity of the model as

$$
v_{c}^{2}(\widetilde{R})=\sum_{n=1}^{m} A_{2 n} \widetilde{R}^{2 n} .
$$

Equation (9) and (12) and using the orthogonality properties of the associated Legendre functions (Arfken \& Weber, 2005), we find that the $A_{2 n}$ constants in (12) and the $C_{2 n}$ constants are related through the expression

$$
C_{2 n}=\frac{4 n+1}{4 n(2 n+1)} \sum_{k=1}^{m} \frac{A_{2 k} I_{k n}}{q_{2 n}(0)},
$$

for $n \neq 0$, where

$$
I_{k n}=\int_{-1}^{1} \eta\left(1-\eta^{2}\right)^{k} P_{2 n}^{\prime}(\eta) d \eta .
$$

This implies that if the constants $A_{2 n}$ are determined by the adjustment of the observational data of the rotation curve, then using (13) we can obtain the values of the constants $C_{2 n}$ which allows us to define the particular thin disc model by means of (7).

From (13) it is easy to see that the value of the constant $C_{0}$ cannot be obtained through this equation. Additionally, from (10) we can see that the surface mass density diverges at the edge of the disc when $\eta=0$, unless the following condition is imposed (Hunter, 1963)

$$
\sum_{n=0}^{m} C_{2 n}(2 n+1) q_{2 n+1}(0) P_{2 n}(0)=0 .
$$

After a couple of operations using the properties of Legendre functions, we arrive at the expression

$$
C_{0}=\sum_{n=1}^{m}(-1)^{n+1} C_{2 n}
$$


by means of which we can obtain the value of $C_{0}$, and furthermore, through (11), calculate the total mass in terms of $A_{2 n}$.

Previously in Gonzáles et al. (2010), it was shown that, in a first approximation, the condition for the stability of the discoidal structure, composed mainly by particles that describe nearly circular motions, is that both epicyclic and vertical frequencies are positive, (Binney \& Tremaine, 2008; Danby, 1988), i.e

$$
\chi^{2}(R)=\left.\frac{\partial^{2} \Phi_{\mathrm{eff}}}{\partial R^{2}}\right|_{z=0}>0,
$$

and

$$
\nu^{2}(R)=\left.\frac{\partial^{2} \Phi_{\mathrm{eff}}}{\partial z^{2}}\right|_{z=0}>0
$$

where

$$
\Phi_{\mathrm{eff}}=\Phi(R, z)+\frac{\ell^{2}}{2 R^{2}},
$$

is the effective potential and $\ell=R v_{c}$ is the specific axial angular momentum.

The procedure used to arrive at the analysis of the epicyclic and vertical frequencies around circular orbits (Binney \& Tremaine, 2008), is valid only for potentials that are smooth or that have at least continuous second derivatives. This means that the analysis of the vertical frequency of disc potentials, such as those analyzed in Gonzáles et al. (2010), is not a reliable indicator of the vertical stability of the corresponding circular orbit. The analysis must take into account the discontinuity in the partial derivative of the potential with respect to $z$ due to the surface distribution of matter in the equatorial plane Viera \& Ramos-caro (2016).

In order to analyze the behavior of the $z$ coordinate of a vertically perturbed orbit in the vicinity of a discoidal distribution, as it was recently pointed out by Viera \& Ramos-caro (2016), we consider an equatorial circular orbit of radius $R$ under the action of a small disturbance, which can be seen as an instantaneous vertical increment $v_{\mathrm{O}}$, in the neighborhood of the particle, which does not affect the value of $\ell$. The equation of movement that allows us to study the temporal evolution of the vertical perturbation is

$$
\ddot{z}=-[2 \Theta(z)-1] \frac{\partial \Phi}{\partial|z|} .
$$

From this equation it can be noted that the vertical disturbance will remain small if and only if $\left.\frac{\partial \Phi}{\partial|z|}\right|_{z=0}>0$. This guarantees that if $v_{0 z}$ is small enough, the perturbed path will oscillate around the original one for a long time. The 
particle will always traverse the disc with a velocity whose vertical component has an absolute value $\left|v_{0 z}\right|$. Then, just after crossing the disc, the particle will also have a velocity with vertical component of magnitude $\left|v_{0 z}\right|$, which means that the movement after crossing the disc will have the same behavior as the movement before crossing it. Therefore, the disturbed orbit will remain oscillating around the original one for a sufficiently small initial vertical velocity.

According to (5), the condition $\left.\frac{\partial \Phi}{\partial|z|}\right|_{z=0}>0$ can be written in terms of the surface mass density $\Sigma$. Then it can be established that in a Newtonian model of a thin disc, a sufficient and necessary condition for vertical stability of a circular orbit of radius $R$ is

$$
\Sigma(\widetilde{R})>0
$$

Since we know that all disc models which attempt to represent realistic distributions of matter satisfy such a condition, then it can be established that equatorial circular orbits in thin disc models are always stable under vertical perturbations.

Therefore, by using then (19) in (17), we can easily obtain the relation

$$
\varkappa^{2}(R)=\frac{1}{R} \frac{d v_{c}^{2}}{d R}+\frac{2 v_{c}^{2}}{R^{2}}
$$

in such a way that, from (12), the epiciclic frequency can be cast as

$$
\tilde{\chi}^{2}(\widetilde{R})=\sum_{n=1}^{m} 2(n+1) A_{2 n} \widetilde{R}^{2 n-2},
$$

where $\tilde{x}=a \varkappa$.

A brief analysis of (22) shows that the obtained models can have radial instabilities near the edge of the disc, because the models are adjusted with the observed rotation curves, which implies that the circular velocity will increase rapidly with the radius and then remain approximately constant. Then, the largest positive value of $d v_{c}^{2} / d R$ occurs at $R=0$ and hence so $\tilde{\varkappa}^{2}$ will have a positive maximum at the center of the disc, and then decrease towards the edge.

\section{Fitting the Rotation Curve}

We will start this section by pointing out the most relevant aspects of the adjustment, which we have previously published in (Gonzales \& Reina, 2016). In order to adjust the previous model to the real observed data, we have taken a sample of spiral galaxies from the Ursa Major cluster. We pick the corresponding data out from Table 4 of the paper by Verheijen \& Sancisi 
(2001), which presents the results of an extensive $21 \mathrm{~cm}$-line synthesis imaging survey of 41 galaxies in the neighborhood of the Ursa Major cluster using the Westerbork Synthesis Radio Telescope. The mean distance between this telescope and the cluster is $18.6 \mathrm{Mpc}$. At this distance, $1 \mathrm{arcmin}$ corresponds to $5.4 \mathrm{kpc}$.

For each rotation curve data, we take as the value of $a$ the value given by the last tabulated radius, i.e. we are assuming that the radius of each galaxy is defined by its corresponding last observed value. Thereafter we take the radii normalized in units of $a$ to fit the rotation curve of every galaxy by means of the model (12). The fits are made through a non-linear least squares fitting using the Levenberg Marquardt algorithm, implemented internally by ROOT (Brun \& Rademakers, 1997) version 5.28, which minimizes the weighted sum of squares of deviations between the fit and the data. We assigned weights to the data points inversely proportional to the square of their errors. These errors correspond to $2 v \Delta v$, being $\Delta v$ the galaxy velocity measurement error.

For each galaxy, we look initially for all the possible fits starting at $m=1$ up to $m=N-1$, with $\mathrm{N}$ the number of measured data pairs $\left(R, v^{2}\right)$, and we take as value for $m$ those for which we get the minimum reduced chi squared $\chi_{r}^{2}$ (the best fit). Now we can discard the galaxies that do not pass the reduced chi squared test (Bevington \& Keith, 2003) with a confidence level of $95 \%$. From the initial 41 galaxies, 26 have survived the reduced chi squared test. These 26 galaxies with their respective information of the fit done are tabulated in Table 1, where the columns 1, 2, 3, 4, 5, 6, 7, and 8 are the name, type of morphology, radius, number of parameters of the fitting function? mass (calculated according to our model), minimum reduced chi squared number of degrees of freedom (equal to $N-1$ ), and the confidence level of the fit, respectively.

Fig. 1 - 4 shown the twenty six adjusted rotation curves of the surviving galaxies. The points with error bars are the observations, as reported in (Verheijen \& Sancisi, 2001), while the solid black line is the fitted rotation curve by using (12), where the values for the $A_{2 n}$ are given by the best fit. As we can see, relation (12) fits quite accurately the observed data of the referred galaxies. Starting from the values obtained for $A_{2 n}$, the values of the constants $C_{2 n}$ are calculated by using the relations (13) and (16). The $C_{2 n}$ constants are tabulated in Table 3. In a similar way, we can use (11) and (16) in order to calculate the mass $\mathscr{M}$ for each galaxy with its respective error; these values are shown together in the fifth column of Table 1. 

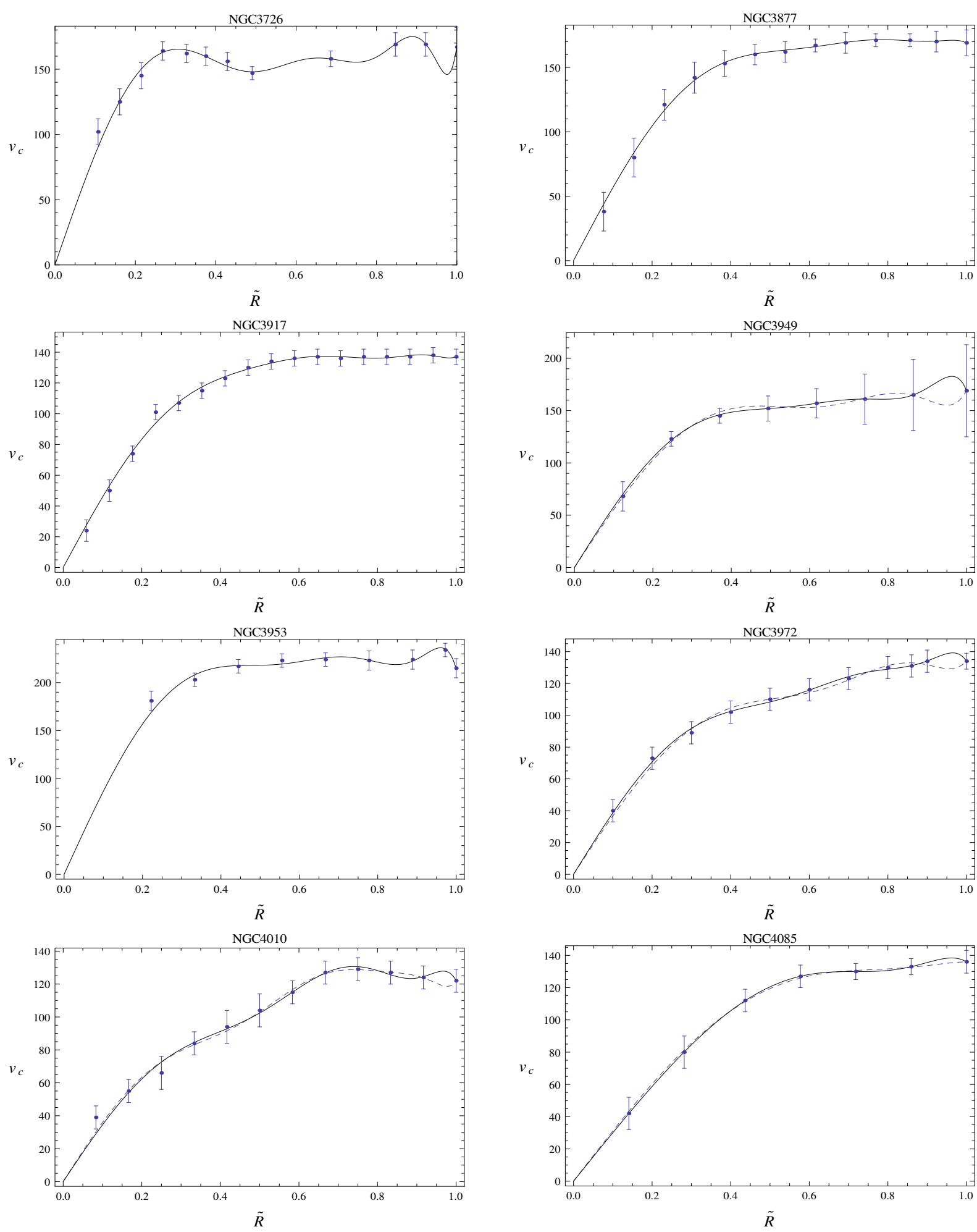

Figure 1. Circular Velocity in $[\mathrm{km} / \mathrm{s}]$ of galaxies NGC3726, NGC3877, NGC3917, NGC3949, NGC3953, NGC3972, NGC4010 and NGC4085. The solid lines represent the rotation curve determined from (12) and the parameters of the best fit listed in Table 1. The dashed lines correspond to the rotation curve for the galaxies radially stable by using the parameters $m$ tabulated in Table 2. The points with error bars are the observations, as reported in (Verheijen \& Sancisi, 2001) 

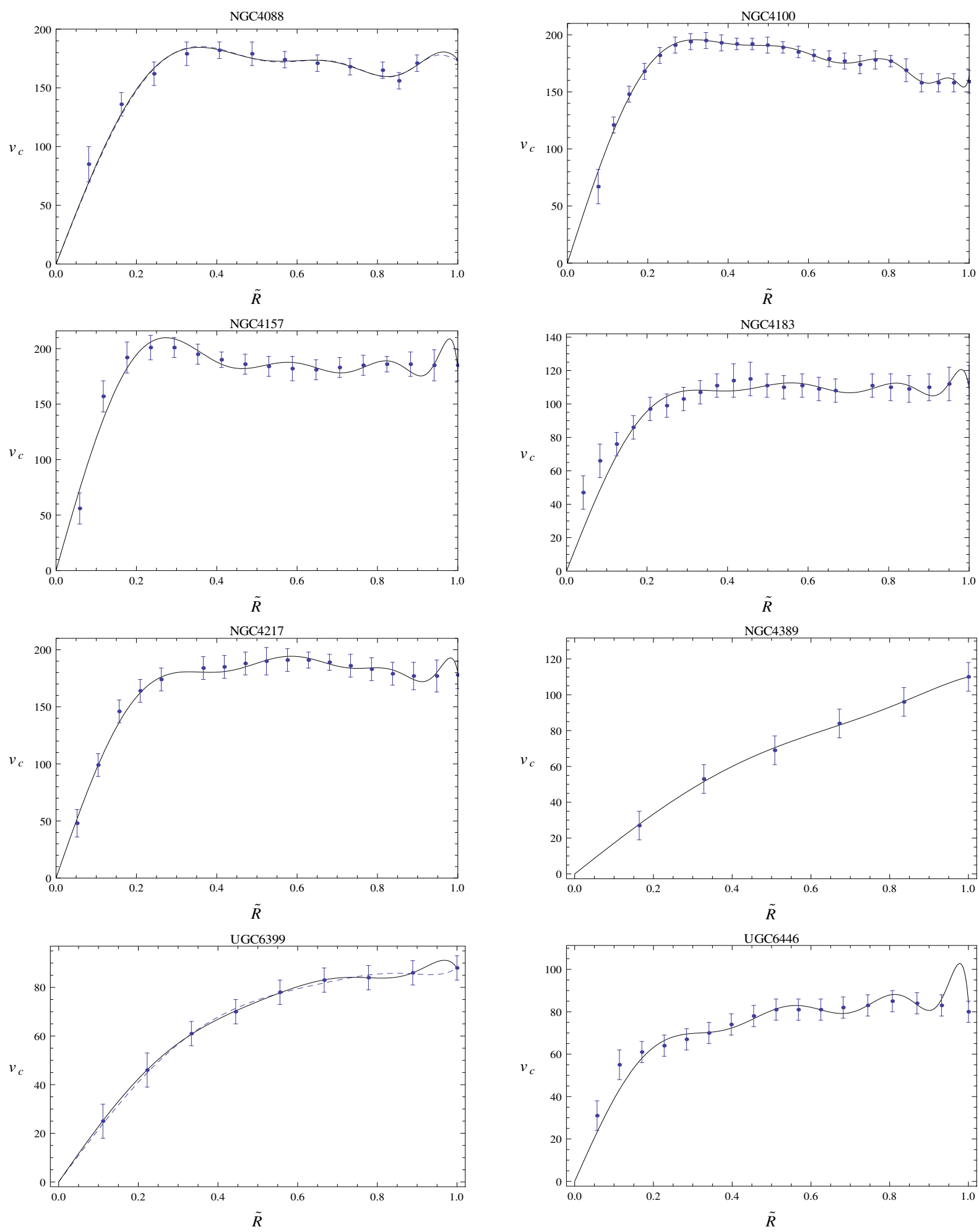

Figure 2. Circular Velocity in [km/s] of galaxies NGC4088, NGC4100, NGC4157, NGC4183, NGC4217, NGC4389, UGC6399 and UGC6446. The solid lines represent the rotation curve determined from (12) and the parameters of the best fit listed in Table 1. The dashed lines correspond to the rotation curve for the galaxies radially stable by using the parameters $m$ tabulated in Table 2. The points with error bars are the observations, as reported in (Verheijen \& Sancisi, 2001) 
Rein et al.

201
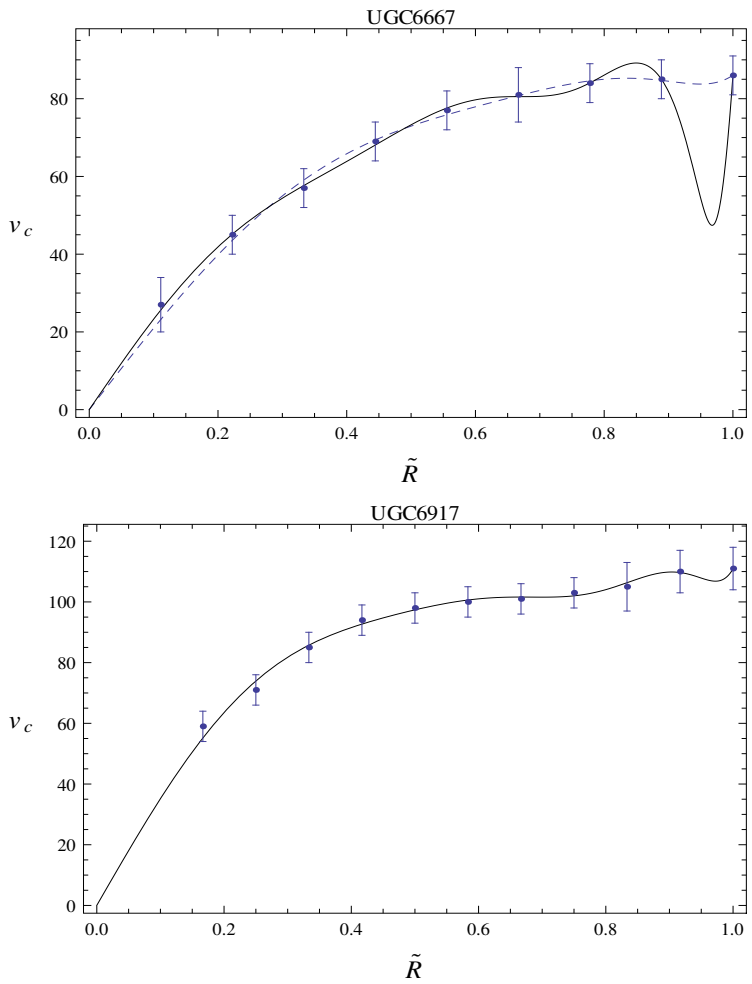

UGC6930

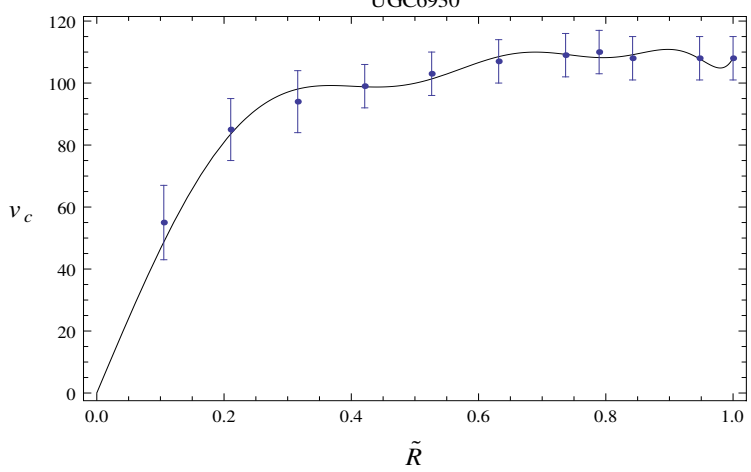

UGC6969

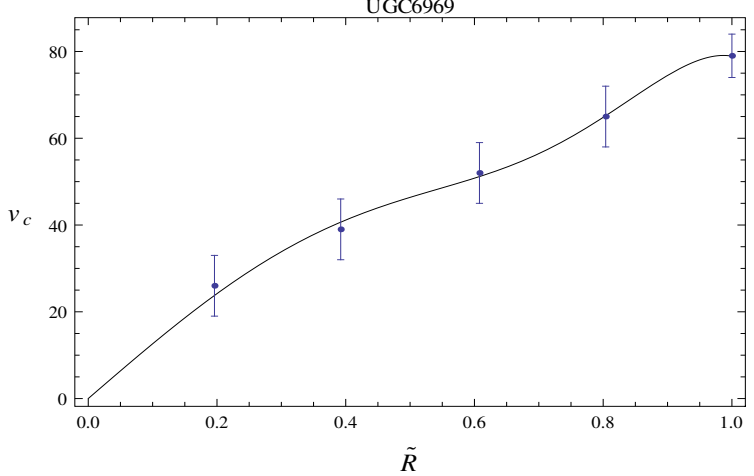

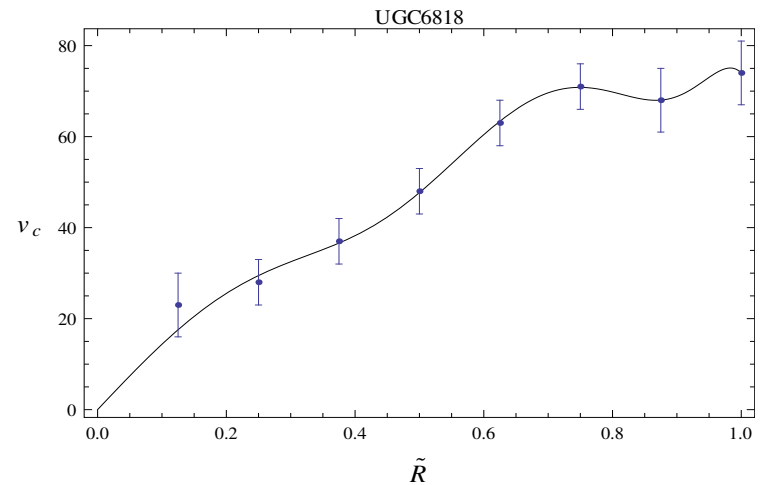
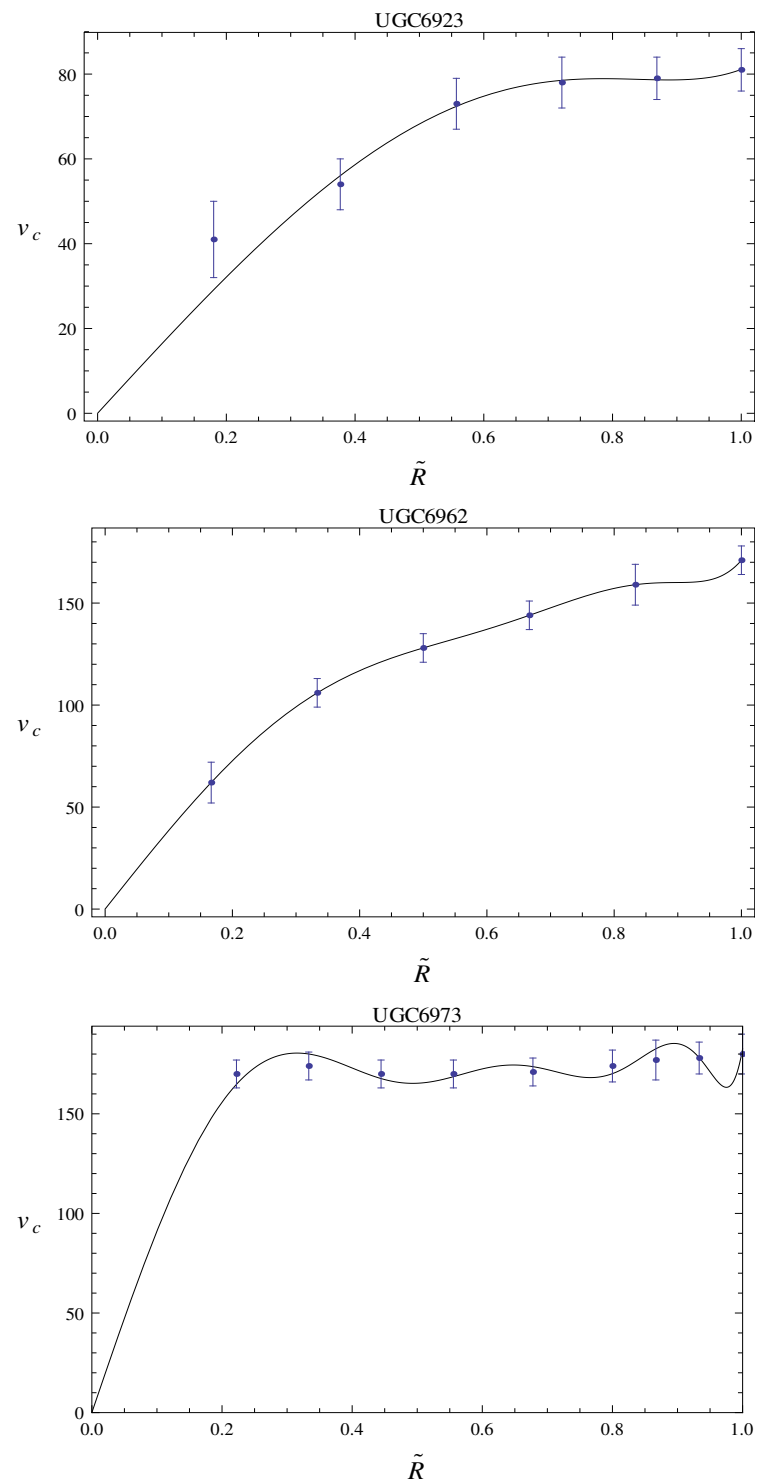

Figure 3. Circular Velocity in [km/s] of galaxies UGC6667, UGC6818, UGC6917, UGC6923, UGC6930, UGC6962, UGC6969 and UGC6973. The solid lines represent the rotation curve determined from (12) and the parameters of the best fit listed in Table 1. The dashed lines correspond to the rotation curve for the galaxies radially stable by using the parameters $m$ tabulated in Table 2. The points with error bars are the observations, as reported in (Verheijen \& Sancisi, 2001)

Universitas Scientiarum Vol. 23 (2): 191-218

http:/ / ciencias.javeriana.edu.co/investigacion/ universitas-scientiarum 

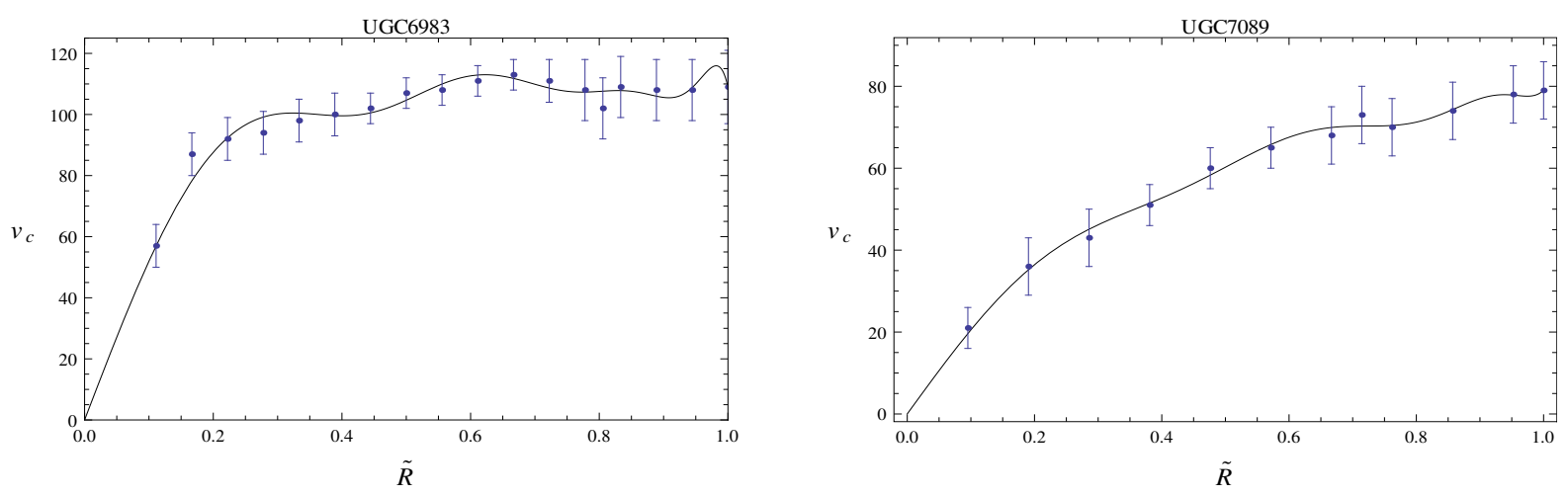

Figure 4. Circular Velocity in [km/s] of galaxies UGC6983 and UGC7089. The solid lines represent the rotation curve determined from (12) and the parameters of the best fit listed in Table 1 . The dashed lines correspond to the rotation curve for the galaxies radially stable by using the parameters $\mathrm{m}$ tabulated in Table 2. The points with error bars are the observations, as reported in (Verheijen \& Sancisi, 2001)

Given that the set of constants $C_{2 n}$ defines completely each particular thin disc model, it is straightforward to compute all the physical quantities characterizing each galaxy. Explicit expressions for the gravitational potential $\Phi(R, z)$ and the surface mass density $\Sigma(R)$ can be easily obtained by using constants $C_{2 n}$ at expressions (7) and (10), they are not presented here. Instead of that, we plot in Fig. 5 - 8 the surface densities for the twenty six galaxies, as functions of the dimensionless radial coordinate $\widetilde{R}=R / a$. The solid black line represents the behavior of the surface mass density that makes use of the parameters obtained by means of the best fit. For the twenty six galaxies we obtain a well behaved surface mass density, having a maximum at the disc center, and hence, decreasing until totally vanishing at the disc's edge. This implies, according to (21), that all galaxies are stable against vertical perturbations.

In a similar way, we can compute the epicyclic frequencies by using (23), and the values of the constants $A_{2 n}$ obtained from the best fit; however, as with the surface mass densities, we will not present the explicit expressions here, and we only show the corresponding plots. Fig. 9 - 12 show with a solid black line the plots of the epicyclic frequencies for the set of galaxies considered. As we can see, seventeen galaxies of the sample have small regions of radial instability close to the disc's edge. For each of these radially unstable galaxies, we consider the possibility that there exists a set of parameters $\bar{m}$, different 
Table 1. Best Fit results of the UMa Cluster.

\begin{tabular}{|c|c|c|c|c|c|c|c|c|}
\hline $\begin{array}{l}\text { Galaxy } \\
\text { (1) }\end{array}$ & $\begin{array}{c}\text { Type } \\
(2)\end{array}$ & $\begin{array}{c}a[\mathrm{kpc}] \\
\text { (3) }\end{array}$ & $\begin{array}{l}\mathrm{m} \\
(4)\end{array}$ & $\begin{array}{r}\mathscr{M}\left[10^{10}\right. \\
\quad(5)\end{array}$ & $\left.\mathscr{M}_{\odot}\right]$ & $\begin{array}{l}\chi_{r}^{2} \\
(\mathbf{6})\end{array}$ & $\begin{array}{r}\text { ndf } \\
(7)\end{array}$ & $\begin{array}{c}\text { C.L \% } \\
(8)\end{array}$ \\
\hline NGC3726 & $\mathrm{SBc}$ & 33.57 & 7 & 12.50 & & 0.4 & 5 & 95 \\
\hline NGC3877 & Sc & 11.7 & 6 & 4.77 & 0.72 & 0.073 & 7 & 95 \\
\hline NGC3917 & Scd & 15.3 & 7 & 4.05 & 0.86 & 0.3 & 10 & 95 \\
\hline NGC3949 & Sbc & 7.29 & 6 & 2.93 & 0.35 & 0.048 & 2 & 95 \\
\hline NGC3953 & $\mathrm{SBbc}$ & 16.2 & 6 & 11.76 & & 0.76 & 3 & 95 \\
\hline NGC3972 & Sbc & 9.0 & 6 & 2.14 & 0.32 & 0.085 & 5 & 95 \\
\hline NGC4010 & $\mathrm{SBb}$ & 10.8 & 6 & 2.30 & 0.93 & 0.36 & 6 & 95 \\
\hline NGC4085 & $\mathrm{Sc}$ & 6.39 & 5 & 1.58 & 0.01 & 0.00015 & 2 & 95 \\
\hline NGC4088 & Sbc & 22.14 & 7 & 9.68 & 3.63 & 0.56 & 6 & 95 \\
\hline NGC4100 & Sbc & 23.49 & 11 & 9.98 & 1.57 & 0.16 & 14 & 95 \\
\hline NGC4157 & $\mathrm{Sb}$ & 30.6 & 8 & 16.10 & & 0.80 & 9 & 95 \\
\hline NGC4183 & Scd & 21.69 & 8 & 3.92 & 2.46 & 0.59 & 15 & 95 \\
\hline NGC4217 & $\mathrm{Sb}$ & 17.19 & 8 & 8.50 & 2.58 & 0.23 & 11 & 95 \\
\hline NGC4389 & $\mathrm{SBbc}$ & 5.49 & 4 & 0.72 & 1.00 & 0.047 & 2 & 95 \\
\hline UGC6399 & $\mathrm{Sm}$ & 8.1 & 6 & 0.83 & 0.04 & 0.0036 & 3 & 95 \\
\hline UGC6446 & $\mathrm{Sd}$ & 15.84 & 8 & 1.74 & 1.09 & 0.30 & 9 & 95 \\
\hline UGC6667 & Scd & 8.1 & 8 & 0.65 & 0.20 & 0.11 & 1 & 95 \\
\hline UGC681 & $\mathrm{Sd}$ & 7.2 & 6 & 0.47 & 0.25 & 0.29 & 2 & 95 \\
\hline UGC6917 & SBd & 10.8 & 7 & 1.71 & 0.71 & 0.28 & 4 & 95 \\
\hline UGC6923 & $\mathrm{Sdm}$ & 5.49 & 4 & 0.46 & 0.43 & 0.47 & 3 & 95 \\
\hline UGC6930 & Sbb & 17.1 & 7 & 2.84 & 0.82 & 0.16 & 4 & 95 \\
\hline UGC6962 & SBcd & 5.4 & 5 & 1.85 & 0.009 & 0.00003 & 1 & 95 \\
\hline UGC6969 & $\mathrm{Sm}$ & 4.59 & 4 & 0.30 & 1.17 & 0.17 & 1 & 95 \\
\hline UGC6973 & Sab & 8.1 & 7 & 3.58 & 2.19 & 1.07 & 2 & 95 \\
\hline UGC6983 & SBcd & 16.2 & 9 & 2.80 & 1.64 & 0.29 & 9 & 95 \\
\hline UGC7089 & $\mathrm{Sdm}$ & 9.45 & 7 & 0.72 & 0.26 & 0.12 & 5 & 95 \\
\hline
\end{tabular}

from those of the best fit (column 4, Table 1), so that the $\chi_{r}^{2}$ test continues to be satisfied with a confidence level of $95 \%$, and which in turn can stabilize some of these galaxies, without altering the physical behavior of the rotation curve and the surface mass density. 

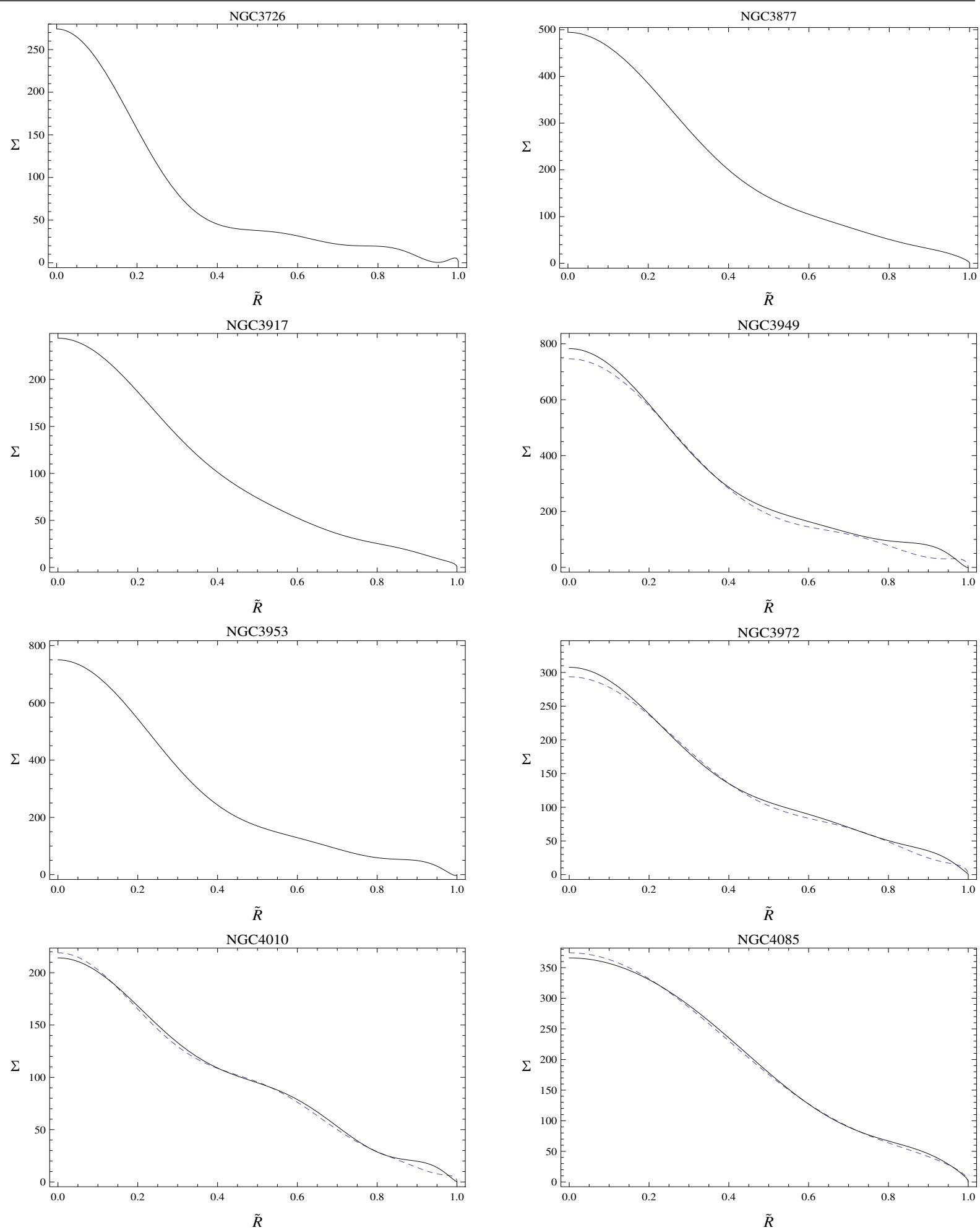

Figure 5. Surface density mass in $\left[\mathscr{M}_{\odot} / \mathrm{pc}^{2}\right]$ for galaxies NGC3726, NGC3877, NGC3917, NGC3949, NGC3953, NGC3972, NGC4010 and NGC4085. The solid lines represent the behavior of the surface density mass determined from (10) and the best fit parameters $m$ listed in Table (1). The dashed lines correspond to the surface density mass for the galaxies radially stable by using the parameters $m$ tabulated in Table (2). For all the galaxies of the sample the surface density has a physically reasonable behavior: maximum at the center of the disc and then decreasing monotonically with the radius. 

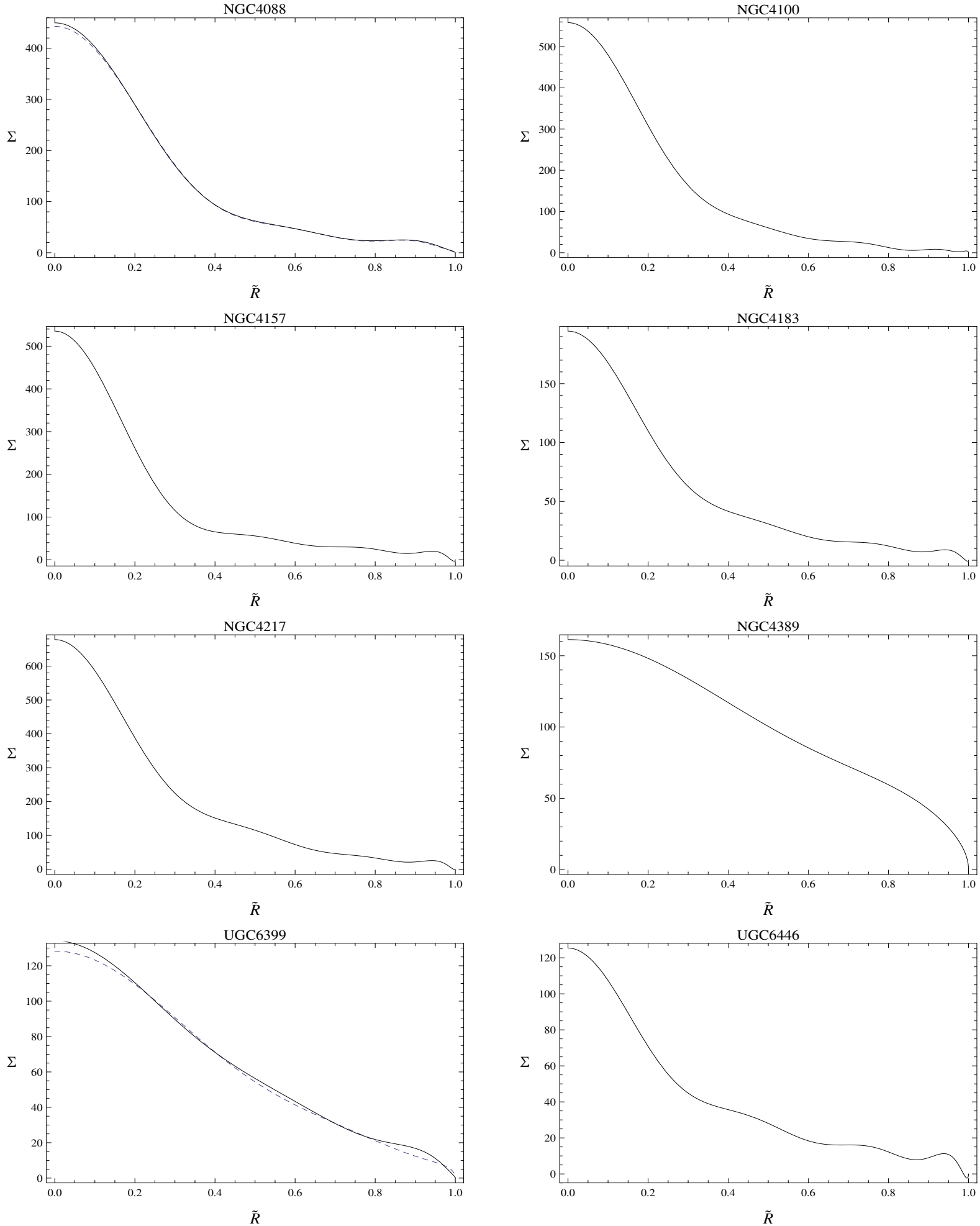

Figure 6. Surface density mass in $\left[\mathscr{M}_{\odot} / \mathrm{pc}^{2}\right]$ for galaxies NGC4088, NGC4100, NGC4157, NGC4183, NGC4217, NGC4389, UGC6399 and UGC6446. The solid lines represent the behavior of the surface density mass determined from (10) and the best fit parameters $m$ listed in Table (1). The dashed lines correspond to the surface density mass for the galaxies radially stable by using the parameters $\mathrm{m}$ tabulated in Table (2). For all the galaxies of the sample the surface density has a physically reasonable behavior: maximum at the center of the disc and then decreasing monotonically with the radius. 

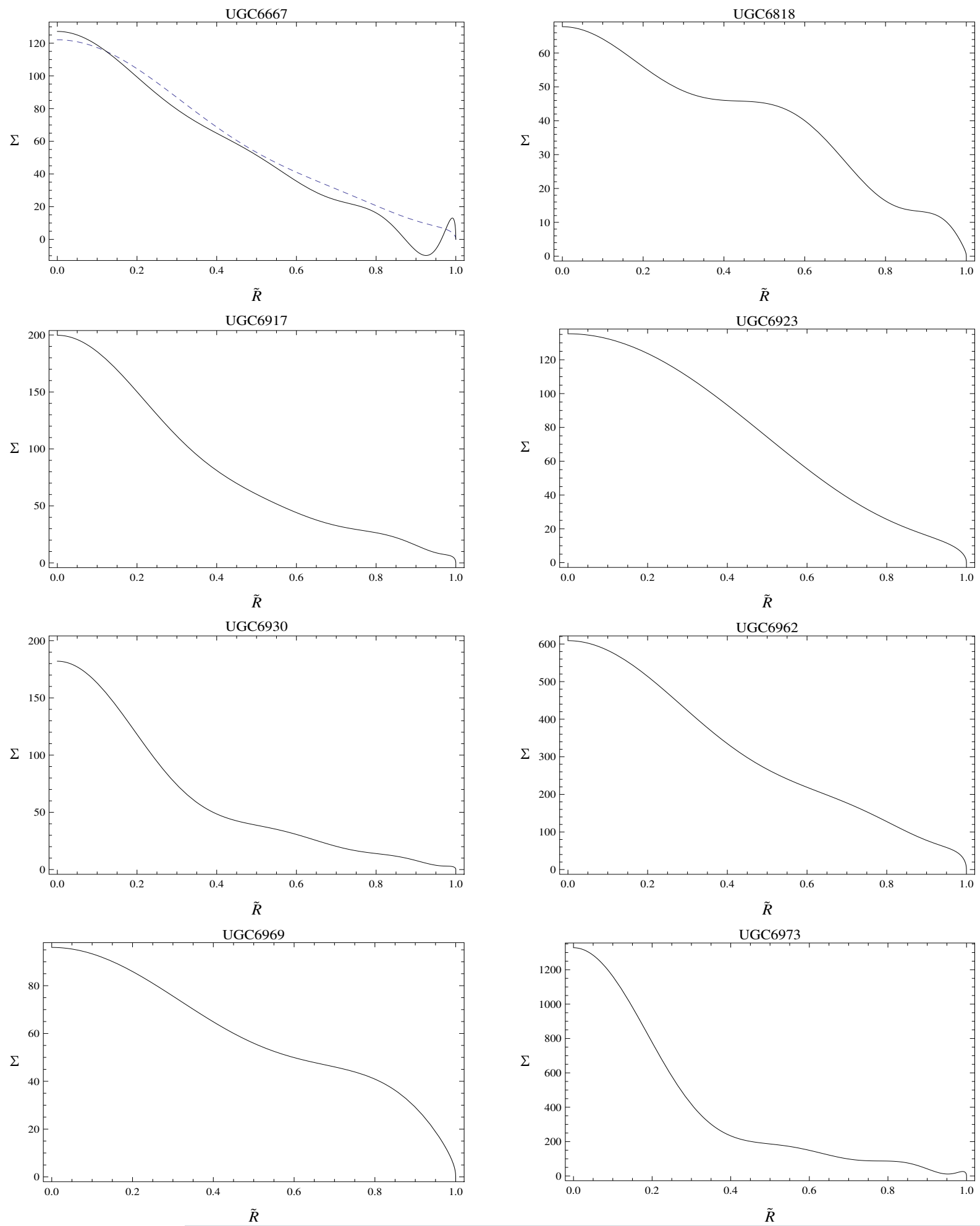

Figure 7. Surface density mass in $\left[\mathscr{M}_{\odot} / \mathrm{pc}^{2}\right]$ for galaxies UGC6667, UGC6818, UGC6917, UGC6923, UGC6930, UGC6962, UGC6969 and UGC6973. The solid lines represent the behavior of the surface density mass determined from (10) and the best fit parameters $m$ listed in Table (1). The dashed lines correspond to the surface density mass for the galaxies radially stable by using the parameters $m$ tabulated in Table (2). For all the galaxies of the sample the surface density has a physically reasonable behavior: maximum at the center of the disc and then decreasing monotonically with the radius. 
Table 2. Stable Galaxies by using the others parameters (different to the best fit ones).

\begin{tabular}{|c|c|c|c|c|c|c|}
\hline $\begin{array}{c}\text { Galaxy } \\
\text { (1) }\end{array}$ & $\begin{array}{l}m \\
(2)\end{array}$ & \multicolumn{2}{|c|}{$\begin{array}{l}\mathrm{m} \\
\text { (3) }\end{array}$} & $\begin{array}{c}x \\
(4)\end{array}$ & $\begin{array}{r}\text { ndf } \\
\text { (5) }\end{array}$ & $\begin{array}{l}\text { C.L } \\
\text { (6) }\end{array}$ \\
\hline NGC3949 & 5 & 2.68 & 0.78 & 0.24 & 3 & 95 \\
\hline NGC3972 & 5 & 2.06 & 0.48 & 0.19 & 6 & 95 \\
\hline NGC4010 & 7 & 2.23 & 0.95 & 0.38 & 5 & 95 \\
\hline NGC4085 & 4 & 1.56 & 1.17 & 0.031 & 3 & 95 \\
\hline NGC4088 & 8 & 9.59 & 4.21 & 0.75 & 5 & 95 \\
\hline UGC6399 & 5 & 0.81 & 0.15 & 0.061 & 4 & 95 \\
\hline UGC6667 & 7 & 0.78 & 0.23 & 0.15 & 4 & 95 \\
\hline
\end{tabular}

In order to guarantee a confidence level of $95 \%$, for each of these galaxies we take a set of parameters $\bar{m}=m \pm 1$. For each of these two values we perform the adjustment, and check whether the instability continues.

If the instability disappears, we have obtained the value of $m$ and the parameters $A_{2 n}$ needed to calculate the mass of the galaxy that stabilizes the model. From this new fit, we manage to stabilize 7 galaxies, keeping the rotation curves and surface mass density with a good behavior. In Fig. 1 - 4, $5-8$, and 9 - 12 we show with dashed lines, the rotation curve, the surface mass density and the epiciclic frequency, respectively, for these 7 galaxies. In Table 2 we show the NGC/UGC name of those galaxies stable after having done the new adjustment.
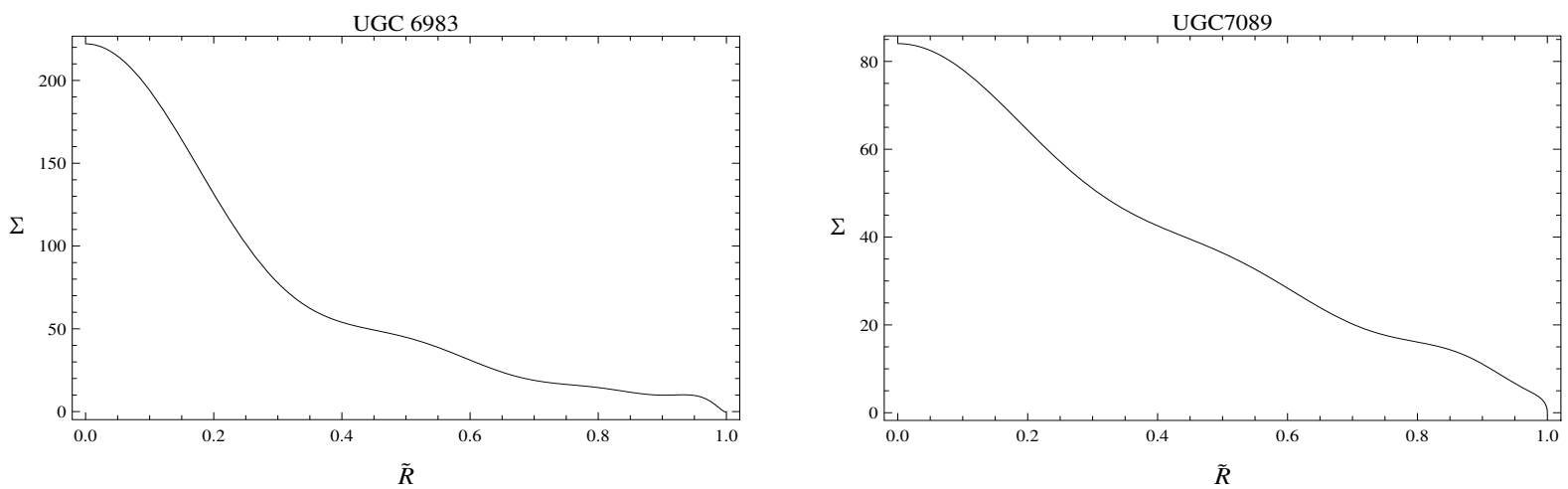

Figure 8. Surface density mass in $\left[\mathscr{M}_{\odot} / \mathrm{pc}^{2}\right]$ for galaxies UGC6983 and UGC7089. The solid lines represent the behavior of the surface density mass determined from (10) and the best fit parameters $m$ listed in Table (1). The dashed lines correspond to the surface density mass for the galaxies radially stable by using the parameters $m$ tabulated in Table (2). For all the galaxies of the sample the surface density has a physically reasonable behavior: maximum at the center of the disc and then decreasing monotonically with the radius. 

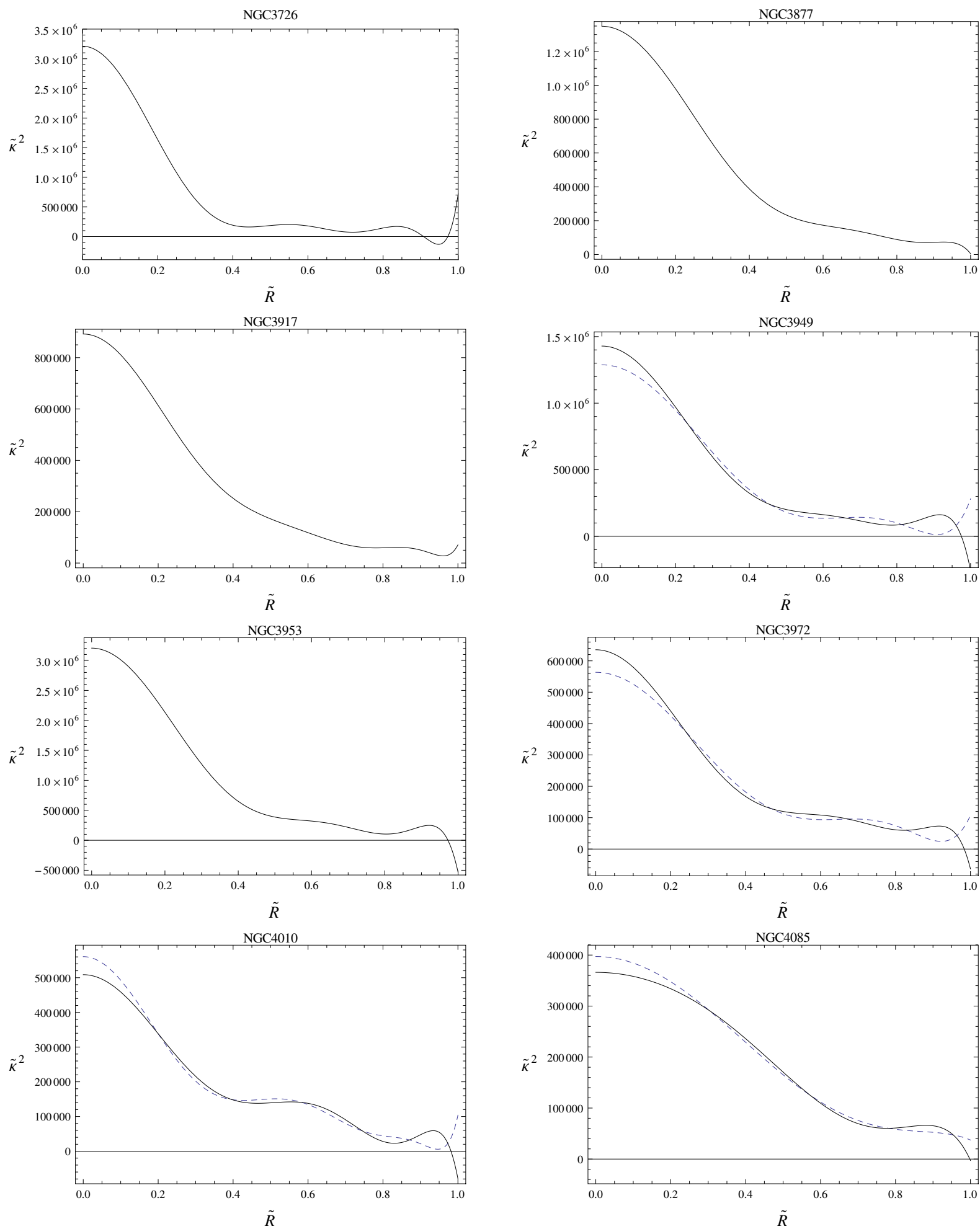

Figure 9. Epicyclic frequency in $\left[\mathrm{Km}^{2} / \mathrm{s}^{2}\right]$ for 26 galaxies NGC3726, NGC3877, NGC3917, NGC3949, NGC3953, NGC3972, NGC4010 and NGC4085. The solid lines represent the epicyclic frequency determined from (23) and the best fit parameters $m$ listed in Table 1 . The regions where the frequency are negative represent zones of the radial instability. The dashed lines correspond to the epicyclic frequency for the galaxies radially stable by using the parameters $m$ tabulated in Table 2 . 

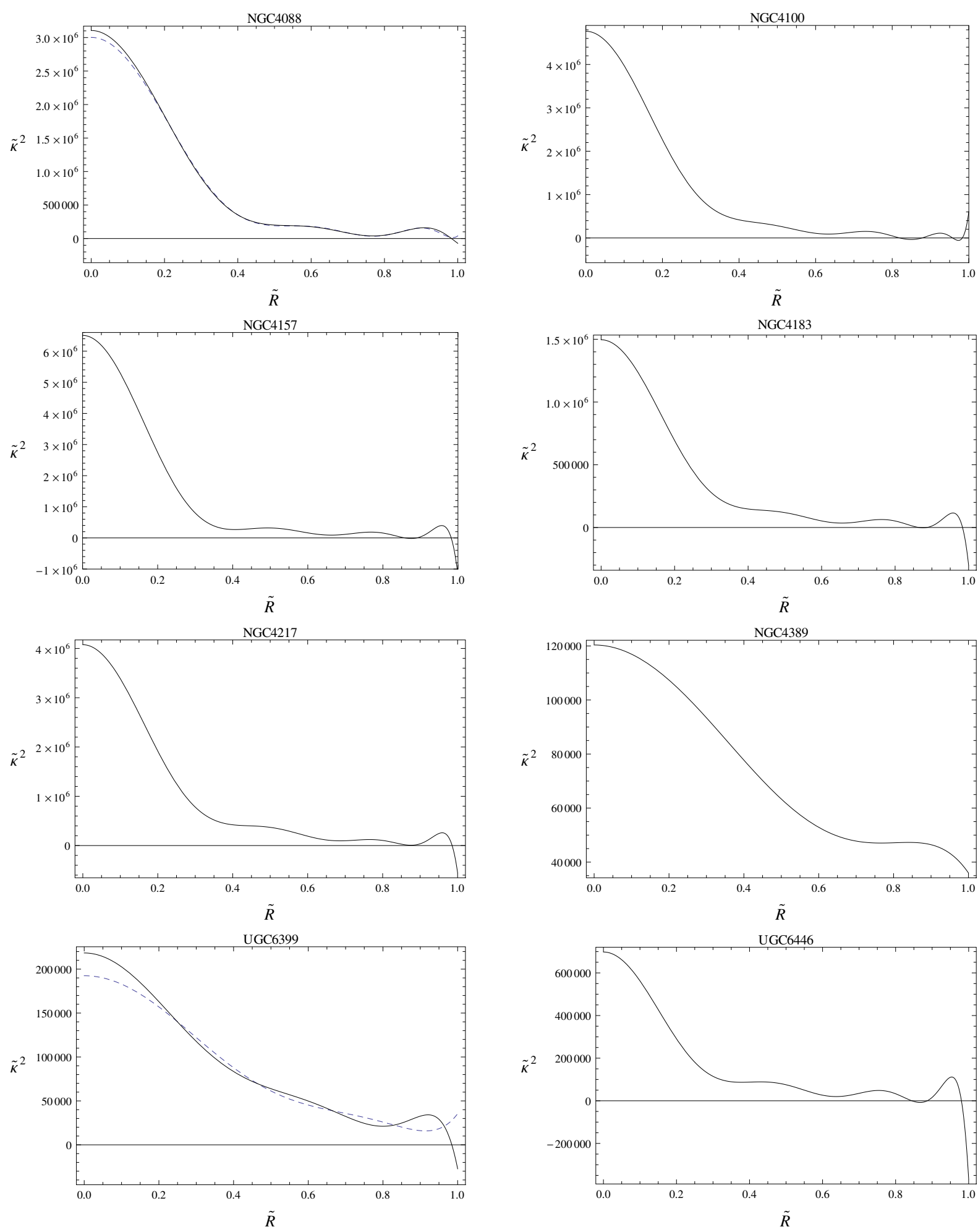

Figure 10. Epicyclic frequency in $\left[\mathscr{K} \mathrm{m}^{2} / \mathrm{s}^{2}\right]$ for 26 galaxies NGC4088, NGC4100, NGC4157, NGC4183, NGC4217, NGC4389, UGC6399 and UGC6446. The solid lines represent the epicyclic frequency determined from (23) and the best fit parameters $m$ listed in Table 1. The regions where the frequency are negative represent zones of the radial instability. The dashed lines correspond to the epicyclic frequency for the galaxies radially stable by using the parameters $m$ tabulated in Table 2 . 

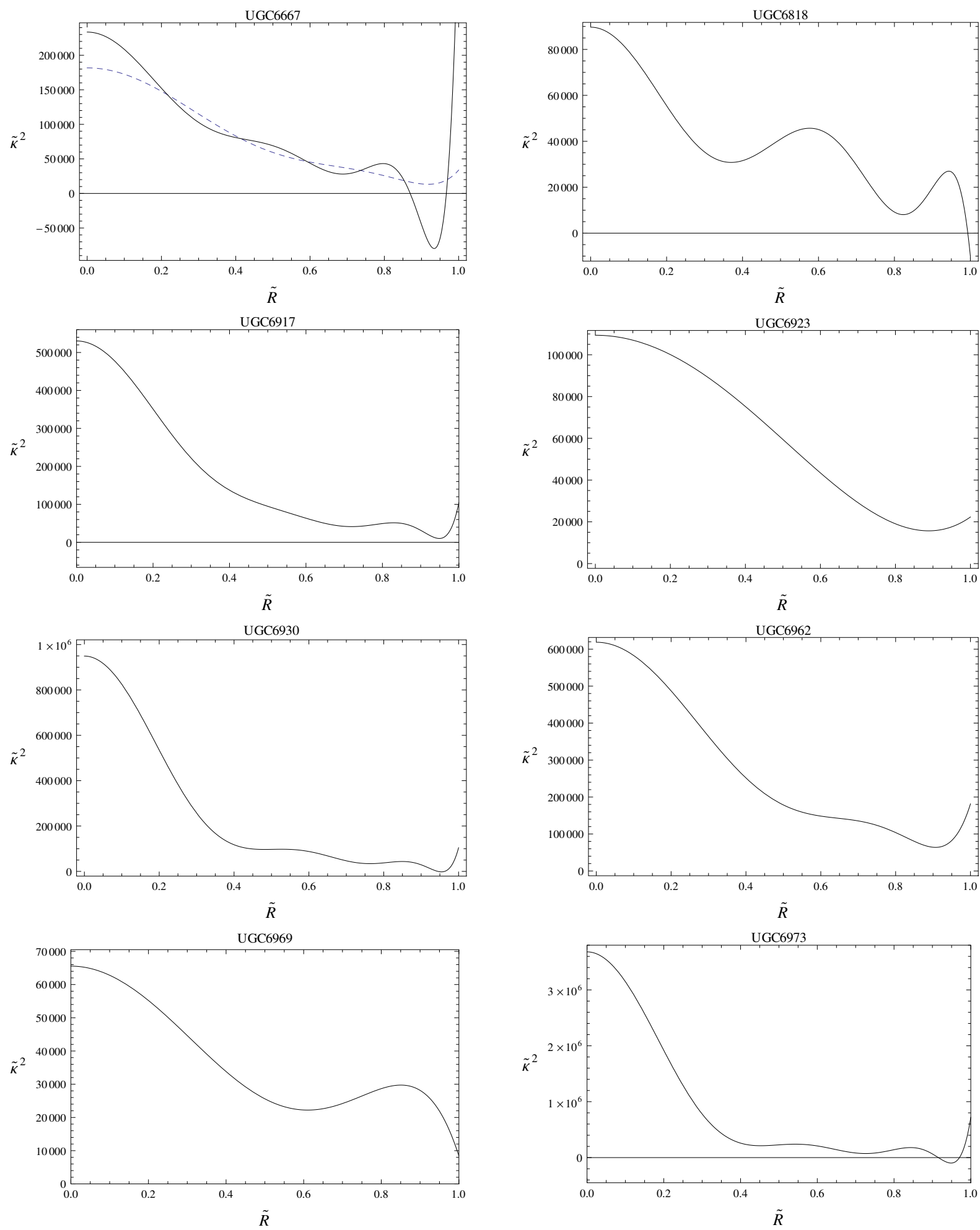

Figure 11. Epicyclic frequency in $\left[\mathscr{K} \mathrm{m}^{2} / \mathrm{s}^{2}\right]$ for 26 galaxies UGC6667, UGC6818, UGC6917, UGC6923, UGC6930, UGC6962, UGC6969 and UGC6973. The solid lines represent the epicyclic frequency determined from (23) and the best fit parameters $m$ listed in Table 1 . The regions where the frequency are negative represent zones of the radial instability. The dashed lines correspond to the epicyclic frequency for the galaxies radially stable by using the parameters $m$ tabulated in Table 2 . 
Table 3. Constants $C_{2 n}\left[\mathrm{~km}^{2} \mathrm{~s}^{-2}\right]$ and values of $m$.

\begin{tabular}{|c|c|c|c|c|c|c|}
\hline & NGC3726 & NGC3877 & NGC3917 & NGC3949 & NGC3953 & NGC3972 \\
\hline$m$ & 7 & 6 & 7 & 6 & 6 & 6 \\
\hline $\mathrm{C}_{0}$ & $16009.9 \pm 8841.7$ & $1755.5 \pm 2643.9$ & $11376.5 \pm 2427.2$ & $17335.5 \pm 2052.82$ & $31237.5 \pm 10660.1$ & $10233.20 \pm 1536.95$ \\
\hline $\mathrm{C}_{2}$ & $25763.0 \pm 6894.5$ & $26696.0 \pm 2148.2$ & $17300.0 \pm 1946.5$ & $24663.8 \pm 1709.1$ & $48537.4 \pm 8903.5$ & $13839.2 \pm 1285.8$ \\
\hline $\mathrm{C}_{4}$ & $16241.8 \pm 4970.8$ & $13960.8 \pm 1418.9$ & $8981.3 \pm 1310.3$ & $12145.7 \pm 1054.6$ & $27895.6 \pm 5435.3$ & $5377.34 \pm 781.57$ \\
\hline $\mathrm{C}_{6}$ & $12510.7 \pm 2301.9$ & $7443.5 \pm 579.6$ & $4572.9 \pm 587.7$ & $7987.6 \pm 409.9$ & $17323.9 \pm 2115.9$ & $2989.12 \pm 302.16$ \\
\hline $\mathrm{C}_{8}$ & $10495.8 \pm 774.7$ & $3938.26 \pm 159.64$ & $2190.76 \pm 195.60$ & $4143.26 \pm 111.31$ & $9537.74 \pm 579.83$ & $1782.92 \pm 81.91$ \\
\hline $\mathrm{C}_{10}$ & $6662.15 \pm 182.66$ & $1854.80 \pm 27.48$ & $1055.87 \pm 46.11$ & $2209.52 \pm 19.35$ & $5608.93 \pm 101.46$ & $1058.89 \pm 14.21$ \\
\hline$C_{12}$ & $4649.38 \pm 27.49$ & $540.78 \pm 2.36$ & $626.81 \pm 6.97$ & $1236.48 \pm 1.68$ & $2799.29 \pm 8.83$ & $493.71 \pm 1.23$ \\
\hline $\mathrm{C}_{14}$ & $2460.97 \pm 2.05$ & & $246.54 \pm 0.52$ & & & \\
\hline & NGC4010 & NGC4085 & NGC4088 & NGC4100 & NGC4157 & NGC4183 \\
\hline$m$ & 6 & 5 & 7 & 11 & 8 & 8 \\
\hline $\mathrm{C}_{0}$ & $9172.97 \pm 3722.18$ & $10629.7 \pm 86.7$ & $18812.6 \pm 7060.2$ & $18295.4 \pm 2879.5$ & $22611.2 \pm 13543.7$ & $7784.60 \pm 4874.35$ \\
\hline $\mathrm{C}_{2}$ & $12969.5 \pm 3085.3$ & $14859.90 \pm 74.07$ & $30409.4 \pm 5657.5$ & $33700.1 \pm 2106.8$ & $36542.3 \pm 10330.7$ & $12261.1 \pm 3728.7$ \\
\hline $\mathrm{C}_{4}$ & $4788.79 \pm 1929.15$ & $5970.22 \pm 42.79$ & $21300.3 \pm 3809.6$ & $26585.90 \pm 1633.84$ & $26514.10 \pm 7684.27$ & $7939.49 \pm 2756.33$ \\
\hline $\mathrm{C}_{6}$ & $1278.83 \pm 755.34$ & $2189.29 \pm 14.0 .3$ & $16026.3 \pm 1724.8$ & $18870.30 \pm 957.27$ & $22312.4 \pm 3896.4$ & $5687.65 \pm 1393.43$ \\
\hline $\mathrm{C}_{8}$ & $782.60 \pm 205.14$ & $226.77 \pm 2.80$ & $9768.51 \pm 575.88$ & $13365.40 \pm 473.25$ & $17330.4 \pm 1509.0$ & $3780.7 \pm 539.7$ \\
\hline $\mathrm{C}_{10}$ & $1162.33 \pm 35.45$ & $-222.52 \pm 0.28$ & $6248.54 \pm 135.79$ & $9053.86 \pm 195.24$ & $13790.01 \pm 38.35$ & $2720.46 \pm 156.82$ \\
\hline $\mathrm{C}_{12}$ & $666.31 \pm 3.06$ & & $3649.31 \pm 20.54$ & $5336.01 \pm 66.03$ & $9112.41 \pm 90.90$ & $1677.83 \pm 32.51$ \\
\hline $\mathrm{C}_{14}$ & & & $846.52 \pm 1.54$ & $3333.74 \pm 17.85$ & $6066.51 \pm 12.13$ & $1288.77 \pm 4.34$ \\
\hline $\mathrm{C}_{16}$ & & & & $2227.11 \pm 3.71$ & $3143.08 \pm 0.80$ & $775.31 \pm 0.29$ \\
\hline $\mathrm{C}_{18}$ & & & & $466.17 \pm 0.56$ & & \\
\hline $\mathrm{C}_{20}$ & & & & $-346.71 \pm 0.05$ & & \\
\hline $\mathrm{C}_{22}$ & & & & $39.000 \pm 0.003$ & & \\
\hline & NGC4010 & NGC4085 & NGC4088 & NGC4100 & NGC4157 & NGC4183 \\
\hline$m$ & 8 & 4 & 6 & 8 & 8 & 6 \\
\hline $\mathrm{C}_{0}$ & $21284.1 \pm 6445.1$ & $5638.36 \pm 7825.18$ & $4422.58 \pm 193.19$ & $4717.08 \pm 2964.13$ & $3433.10 \pm 1059.35$ & $2819.11 \pm 1498.49$ \\
\hline $\mathrm{C}_{2}$ & $34763.6 \pm 4996.3$ & $6431.37 \pm 6686.24$ & $6069.45 \pm 166.24$ & $6619.21 \pm 2253.93$ & $5623.37 \pm 843.24$ & $3462.67 \pm 1231.46$ \\
\hline $\mathrm{C}_{4}$ & $23185.5 \pm 3591.8$ & $1170.86 \pm 3949.43$ & $2405.43 \pm 104.02$ & $3385.34 \pm 1693.72$ & $2626.65 \pm 570.67$ & $687.04 \pm 791.07$ \\
\hline $\mathrm{C}_{6}$ & $15182.8 \pm 1779.8$ & $538.56 \pm 3949.43$ & $1049.56 \pm 40.54$ & $2335.25 \pm 849.31$ & $500.23 \pm 272.06$ & $-103.66 \pm 309.71$ \\
\hline $\mathrm{C}_{8}$ & $9590.53 \pm 682.73$ & $160.70 \pm 98.04$ & $349.84 \pm 10.99$ & $1361.56 \pm 326.45$ & $635.99 \pm 102.82$ & $-52.92 \pm 8393$ \\
\hline $\mathrm{C}_{10}$ & $7471.16 \pm 197.7$ & & $235.64 \pm 1.90$ & $1142.27 \pm 94.52$ & $133.47 \pm 29.73$ & $300.78 \pm 14.53$ \\
\hline $\mathrm{C}_{12}$ & $5101.2 \pm 41.0$ & & $176.79 \pm 0.16$ & $700.69 \pm 19.58$ & $22.83 \pm 6.19$ & $206.56 \pm 1.26$ \\
\hline $\mathrm{C}_{14}$ & $3514.80 \pm 5.48$ & & & $693.49 \pm 2.61$ & $399.50 \pm 0.83$ & \\
\hline $\mathrm{C}_{16}$ & $1771.08 \pm 0.36$ & & & $625.54 \pm 0.17$ & $-62.00 \pm 0.05$ & \\
\hline
\end{tabular}




\begin{tabular}{|ccccccc}
\hline & UGC 6917 & UGC 6923 & UGC 6930 & UGC 6962 & UGC 6969 & UGC 6973 \\
\hline$m$ & 7 & 4 & 6 & 5 & 4 & 7 \\
\hline$C_{0}$ & $6817.49 \pm 2827.27$ & $3643.81 \pm 3385.88$ & $7138.06 \pm 2053.24$ & $14703.70 \pm 70.36$ & $2581.43 \pm 11011.10$ & $18992.0 \pm 11644.7$ \\
\hline$C_{2}$ & $9872.18 \pm 2247.10$ & $5050.79 \pm 3136.25$ & $11148.6 \pm 1673.2$ & $19237.60 \pm 60.21$ & $3060.25 \pm 9391.91$ & $30669.5 \pm 9338.3$ \\
\hline$C_{4}$ & $4747.13 \pm 1550.73$ & $1848.4 \pm 1264.4$ & $6259.85 \pm 1078.51$ & $6566.63 \pm 34.57$ & $379.40 \pm 5581.83$ & $19946.6 \pm 6281.2$ \\
\hline$C_{6}$ & $2679.53 \pm 695.11$ & $441.42 \pm 171.24$ & $3852.20 \pm 476.19$ & $2876.26 \pm 11.18$ & $371.72 \pm 1363.41$ & $15086.20 \pm 2828.47$ \\
\hline$C_{8}$ & $1432.15 \pm 230.35$ & $2831.9 \pm 18012.4$ & $2757.99 \pm 158.01$ & $1605.40 \pm 2.26$ & $201.15 \pm 140.05$ & $11701.9 \pm 943.9$ \\
\hline$C_{10}$ & $586.12 \pm 54.17$ & & $1241.35 \pm 5.67$ & $761.86 \pm 0.23$ & & $7538.67 \pm 222.53$ \\
\hline$C_{12}$ & $386.02 \pm 8.19$ & & $493.56 \pm 0.43$ & & & $5230.59 \pm 33.62$ \\
\hline$C_{14}$ & $244.96 \pm 0.61$ & & & & & $2576.66 \pm 2.52$ \\
\hline & NGC4010 & NGC4085 & NGC4088 & NGC4100 & NGC4157 & NGC4183 \\
\hline$m$ & 9 & 7 & & & & \\
\hline$C_{0}$ & $7449.42 \pm 4350.24$ & $3262.94 \pm 1169.42$ & & & & \\
\hline$C_{2}$ & $11569.70 \pm 3213.94$ & $4286.84 \pm 932.78$ & & & & \\
\hline$C_{4}$ & $6962.65 \pm 2516.34$ & $1471.84 \pm 637.70$ & & & & \\
\hline$C_{6}$ & $4434.89 \pm 1368.96$ & $649.82 \pm 285.19$ & & & & \\
\hline$C_{8}$ & $2800.37 \pm 589.07$ & $282.10 \pm 94.8$ & & & & \\
\hline$C_{10}$ & $2349.54 \pm 198.74$ & $206.46 \pm 22.37$ & & & & \\
\hline$C_{12}$ & $1742.34 \pm 50.98$ & $256.09 \pm 3.39$ & & & & \\
\hline$C_{14}$ & $1051.95 \pm 9.40$ & $129.85 \pm 0.25$ & & & & \\
\hline$C_{16}$ & $361.66 \pm 1.12$ & & & & & \\
\hline$C_{18}$ & $-89.63 \pm 0.07$ & & & & & \\
\hline
\end{tabular}

The number of parameters $m$ used in this new fit are shown in column (2). In columns (4) and (5) we show the reduced chi square and the number of degrees freedom, respectively. In (3) we present the mass values in order to obtain galaxies radially stable.

\section{Concluding Remarks}

We have presented a mass model for a set of twenty six spiral galaxies of the Ursa Major Cluster. The model is adjusted very precisely with the data of the rotation curve of the galaxies of the sample, considering that all the mass is concentrated only in the stellar disc. In this way the model implies that it is not always necessary to introduce a halo of dark matter or MOND theories in order to describe properly the rotation curve of the galaxies. In all cases the best statistical fit between the data and the model is fixed to a confidence level of $95 \%$. As was mentioned by Gonzáles et al. (2010), the equation (12) can be considered as a kind of "universal rotation curve" for flat galaxies, which can easily be adjusted to the observed data of the rotation curve of any particular spiral galaxy. 

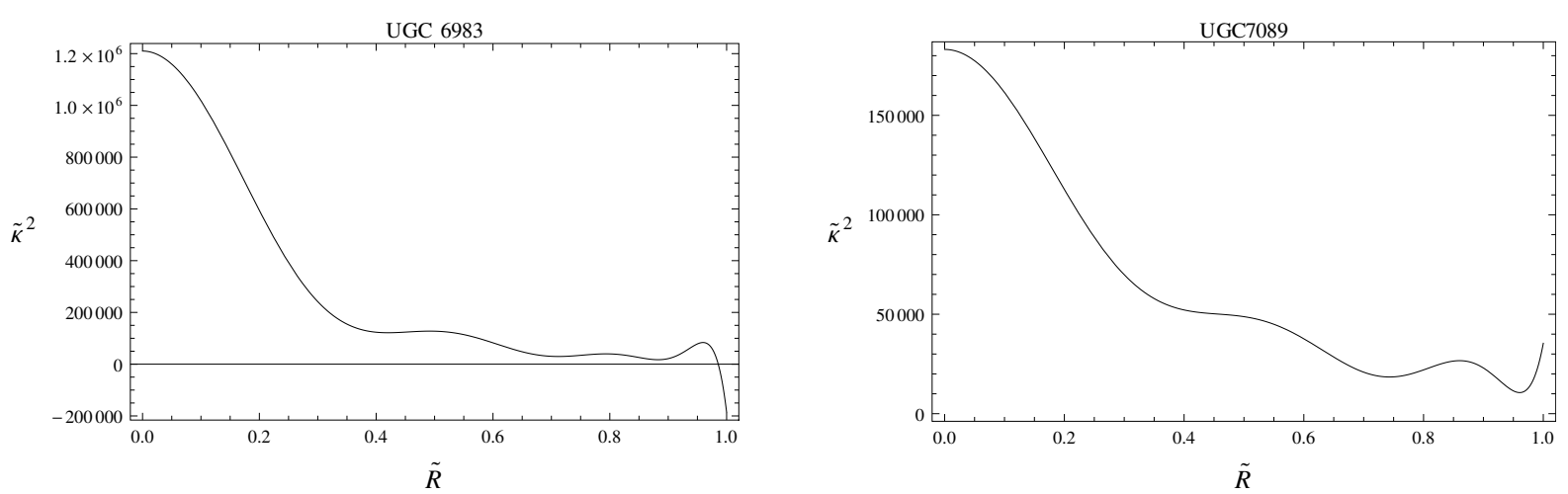

Figure 12. Epicyclic frequency in $\left[\mathscr{K} \mathrm{m}^{2} / \mathrm{s}^{2}\right]$ for 26 galaxies for galaxies UGC6983 and UGC7089. The solid line represent the epicyclic frequency determined from (23) and the best fit parameters $m$ listed in Table 1 . The regions where the frequency are negative represent zones of the radial instability. The dashed line correspond to the epicyclic frequency for the galaxies radially stable by using the parameters $m$ tabulated in Table 2.

The surface mass density of each galaxy present a good behavior: maximum at the center and vanishing at the edge, which is in concordance with the observed luminosity profile of many spiral galaxies, and the obtained values for the corresponding total mass $\mathscr{M}$ it agrees with the expected order of magnitude. Besides, considering the stability criterion constructed by Vieira \& Ramos-Caro (2016), and taking into account that all of the mass density profiles obtained here are positive, we can conclude that all the models are vertically stable, contrary to the statements shown in Gonzáles et al. (2010). Thus, we remark that our formalism is a powerful method to obtain stable maximum disc describing the optical region of many HSB galaxies.

On the other hand, the analysis of the epicyclic frequency reveals that 17 galaxies of the sample present small regions of radial instability near the disc edge. From the equation (23) was expected that this unstable behavior depends only of the fit parameters $A_{2 n}$. We have analyzed the behavior of the radial frequency for all the possible values of $m$, different to those given by the best fit, but so that the confidence level remains at $95 \%$, and thus, we have achieved that 7 of the 17 unstable galaxies present a stable behavior.

According to the results obtained, we consider that the models presented can be considered as a first approximation for obtaining more realistic models. We particularly believe that the mass values $\mathscr{M}$ of the 26 galaxies modeled can be taken as an estimate of the upper limit for the mass of the disc of these galaxies, since in the models it was considered that the whole mass was concentrated in the galactic disc. 


\section{Acknowledgements}

This research was partially supported by VIE-UIS, under grants number 1838 and 2314, and COLCIENCIAS, Colombia, under grant number 8840. JIR and FLS wants to thank the support from Centro de Investigaciones, Universidad Santo Tomás, Bucaramanga.

\section{Conflict of interest}

None.

\section{References}

Arfken G, Weber H. Mathematical Methods for Physicists, 6th Ed., Academic Press, 2005.

Bajkova AT, Bobylev VV. Rotation curve and mass distribution in the galaxy from the velocities of objects at distances up to $200 \mathrm{kpc}$, Astronomy Letters, 42(49): 567-582, 2016.

doi: 10.1134/S1063773716090012

Bateman H. Partial Differential Equations, First American Edition, New York, Dover, 1944.

Bevington P, Keith D. Data Reduction and Error Analysis for the Physical Sciences, 3rd Ed., Mc Graw Hill. 2003.

Binney J, Tremaine S. Galactic Dynamics, 2nd Ed., Princeton University Press. 2008.

Brun R, Rademakers F. ROOT - An object oriented data analysis framework, Nuclear Instruments and Methods in Physics Research A, 389(1): 81-86, 1997.

doi: 10.1016/S0168-9002(97)00048-X

Danby JMA. An Introduction to Stellar Astrophysics, Wiley. 1988.

González GA, Plata-Plata S, Ramos-Caro J. Finite thin disc models of four galaxies in the Ursa Major cluster: NGC3877, NGC3917, NGC3949 and NGC4010, Monthly Notices of the Royal Astronomical Society, 404(1): 468-474, 2010.

doi: 10.1111/j.1365-2966.2010.16303.x

González GA, Reina JI. An infinite family of generalized Kalnajs disks, Monthly Notices of the Royal Astronomical Society, 371(4): 1873-1876, 2006.

doi: 10.1111/j.1365-2966.2006.10819.x 
González GA, Reina JI. Analytical Potentials for Flat Galaxies with Spheroidal Halos. Revista de la Academia Colombiana de Ciencias Exactas Físicas y Naturales, 40(156): 402-411, 2016.

doi: $10.18257 /$ raccefyn.376

Hunter C. The structure and stability of self-gravitating disks, Monthly Notices of the Royal Astronomical Society, 126(4): 299-315, 1963.

doi: $10.1093 /$ mnras/126.4.299

Kalnajs AJ. Mass distribution and dark halos: Dussion, in: Internal kinematics and dynamics of galaxies, Proceedings of the IAU Symposium, Dordrecht, D. Reidel Publishing Co., 87-88, 1983.

Kent SM. Dark matter in spiral galaxies. I - Galaxies with optical rotation curves, The Astronomical Journal, 91(6): 1301-1327, 1986.

doi: $10.1086 / 114106$

LeBlanc F. Galactic Dynamics, 2nd Ed., Princeton University Press. 2010.

Oort JH. Observational evidence confirming Lindblad's hypothesis of a rotation of the galactic system, Bulletin of the Astronomical Institutes of the Netherlands, 3(120): 275-282, 1927.

Palunas P, Williams TB. Maximum disc mass models for spiral galaxies, The Astronomical Journal, 120(6): 2884-2903, 2000.

doi: $0.1086 / 316878$

Rubin VC, Burstein D, Ford WK, Thonnard N. Rotation Velocities of $16 \mathrm{SA}$ Galaxies and a Comparison of Sa, Sb, and SC Rotation Properties, The Astrophysical Journal, 289(1): 81-104, 1985.

doi: $10.1086 / 162866$

Sofue Y, Honma M, Omodaka T. Unified Rotation Curve of the Galaxy - Decomposition into de Vaucouleurs Bulge, disc, Dark Halo, and the 9-kpc Rotation Dip, Publications of the Astronomical Society of Japan, 61(2): 227-236, 2009.

doi: $10.1093 / \mathrm{pasj} / 61.2 .227$

Van Albada TS, Bahcall JN, Begeman K, Sancisi R. Distribution of dark matter in the spiral galaxy NGC 3198, The Astrophysical Journal, 295(1): 305-313, 1985.

doi: $10.1086 / 163375$ 
Verheijen MAW, Sancisi R. The Ursa Major cluster of galaxies. IV. HI synthesis observations, Astronomy and Astrophysics, 370(3): 765-867, 2001.

doi: 10.1051/0004-6361:20010090

Vieira RSS, Ramos-Caro J. Integrability of motion around galactic razorthin disks, Celestial Mechanics and Dynamical Astronomy, 126(4): 483-500, 2016.

doi: 10.1007/s10569-016-9705-0 


\section{Masa de galaxias espirales por medio de un modelo de disco máximo}

Resumen. Presentamos modelos de masa de disco máximo para un conjunto de galaxias espirales del Cluster Ursa Major. Los modelos se obtienen por medio del método de Hunter y las soluciones particulares se eligen de tal manera que las velocidades circulares se ajustan muy exactamente a las curvas de rotación observadas de algunas galaxias espirales específicas. Bajo la hipótesis del disco máximo, suponemos que la masa del disco es lo más grande posible, en consonancia con la curva de rotación de la galaxia. Por lo tanto, la contribución de la masa del halo de la materia oscura se considera insignificante en las partes internas de las espirales. Los modelos reproducen la estructura general de las curvas de rotación en la mayoría de las galaxias, proporcionando buenos ajustes para calcular la masa total de estas galaxias obteniendo valores del orden de $10^{10} \mathscr{M}_{\odot}$. Basados en el criterio de estabilidad vertical presentado por Vieira and Ramos-Caro (2016), encontramos que todas las galaxias analizadas presentan un comportamiento verticalmente estable. Por otro lado, a partir del análisis de la frecuencia epicíclica se observa que todos los modelos presentaron mayormente un comportamiento estable radial excepto en el borde del disco.

Palabras clave: Teoría del potencial; Dinámica Estelar; Masa de Galaxias.

\section{Massa de galáxias espirais por meio de um modelo de disco máximo}

Resumo. Apresentamos modelos de massa de disco máximo para um conjunto de galáxias espirais do Cluster Ursa Major. Os modelos são obtidos por meio do método Hunter e as soluções particulares são escolhidas de tal forma que as velocidades circulares são ajustadas com muita precisão às curvas de rotação observadas de algumas galáxias espirais específicas. Sob a hipótese de disco máximo, supomos que a massa do disco é tão grande quanto possível, consistente com a curva de rotação da galáxia. A contribuição de massa do halo da matéria escura é, portanto, assumida como insignificante nas partes internas das espirais. Os modelos reproduzem a estrutura geral das curvas de rotação na maioria das galáxias, proporcionando bons ajustes para calcular a massa total dessas galáxias obtendo valores da ordem de $10^{10} \mathscr{M}_{\odot}$. Com base no critério de estabilidade vertical apresentado por Vieira and Ramos-Caro (2016), descobrimos que todas as galáxias analisadas apresentam um comportamento verticalmente estável. Por outro lado, a partir da análise da frequência epicíclica, descobrimos que todos os modelos apresentaram principalmente um comportamento estável radial, exceto na borda do disco.

Palavras-chave: Teoria do Potencial; Dinâmica estelar; Massa Galáctica. 


\section{Jerson I. Reina}

He is a professor full time of mathematics and physics at the Universidad Santo Tomás. The author obtained his PhD degree in Physics at Universidad Industrial de Santander. His main research areas are astrophysics and galactic dynamics.

\section{Framsol López-Suspes}

His is PhD in Natural Sciences - Physics of the Universidad Industrial de Santander. He is currently a professor at the Universidad Santo Tomás in the Department of Basic Sciences. His subjects of interest are dynamical systems, dynamics of galaxies, and general relativity.

\section{Guillermo A. González}

He is Professor of Physics at Universidad Industrial de Santander. He obtained his PhD degree in Applied Mathematics at Universidade Estadual de Campinas. His research areas are Astrophysics, General Relativity and Mathematical Physics. 